\title{
Systemic Inflammasome Activation and Pyroptosis Associate with the Progression of Alzheimer's Disease
}

wenjuan rui

Nanjing Medical University Affiliated Brain Hospital: Nanjing Brain Hospital https://orcid.org/00000002-0185-2091

\section{Hong Xiao}

Nanjing Brain Hospital

Yi Fan

Nanjing Brain Hospital

\section{Zhongxuan Ma}

Nanjing Brain Hospital

\section{Ming Xiao}

Nanjing Medical University

Sheng Li ( $\nabla$ shengli_116@163.com )

Nanjing Brain Hospital

Jingping Shi

Nanjing Brain Hospital

\section{Research}

Keywords: Alzheimer's disease, Gasdermin D, Neuroinflammation, Inflammasome, Pyroptosis, Interleukin$1 \beta$

Posted Date: May 24th, 2021

DOI: https://doi.org/10.21203/rs.3.rs-502155/v1

License: (c) (i) This work is licensed under a Creative Commons Attribution 4.0 International License. Read Full License 


\section{Abstract}

Background: Growing evidence indicates that inflammasome-mediated inflammation plays an important role in the pathophysiology of Alzheimer's disease (AD). Likewise, gasdermin D (GSDMD) as executive molecule in inflammasome-induced pyroptosis is also involved in many neurological disorders. However, it is not clear whether inflammasome and pyroptosis is activated in the periphery of AD patients and influences central inflammation. The aim of this study was to evaluate the association between systemic inflammasome-induced pyroptosis and clinical features in the progression of $A D$.

Methods: $A$ total of 86 participants, including 33 patients with AD, 33 patients with amnestic mild cognitive impairment (aMCl), and 20 controls, were included in this study. The cognitive level of each participant was evaluated, including Mini-mental State Examination (MMSE) and Montreal Cognitive Assessment (MoCA) scores were assigned. We collected blood samples from each participant. Gene transcriptomes of peripheral blood mononuclear cells (PBMCs) were determined by RNA-seq. The expression levels of inflammasome-related genes/proteins in PBMCs were determined using quantitative polymerase chain reaction and western blotting. Cerebrospinal fluid (CSF) samples were collected from all AD patients. The levels of IL-1 $\beta, A \beta 1-42$, p-tau, and t-tau in CSF, as well as the plasma IL-1 $\beta$ level, were measured by enzyme-linked immunosorbent assay. Lastly, a low dose of lipopolysaccharides (LPS) was performed to investigate the effects of systemic pyroptosis in AD mice model.

Results: Several genes involved in the inflammatory response pathway were enriched in PBMCs of $A D$ patients. The mRNA and protein levels of NLRP3, caspase-1, GSDMD, and IL-1 $\beta$ were all increased in PBMCs from $A D$ and $\mathrm{aMCl}$ patients. The IL-1 $\beta$ levels in plasma and CSF in $A D$ and $\mathrm{aMCl}$ patients were significantly higher than those in controls and have a negative correlation with levels of A $1-42$ in CSF, MMSE and MOCA scores. Furthermore, there was a positive correlation between the IL-1 $\beta$ level in plasma and $C S F$ of $A D$ or aMCl patients. In addition, animal experiments also showed that systemic pyroptosis aggravates neuroinflammation in 5×FAD mice.

Conclusions: All these findings showed that the canonical inflammasome pathway and GSDMD-induced pyroptosis is activated in PBMCs from $A D$ and aMCl patients. Proinflammatory cytokine IL- $1 \beta$ in periphery is highly associated with the pathological process of AD. Targeting peripheral inflammasomes and pyroptosis may be a strategy to inhibit neuroinflammation in $A D$.

\section{Introduction}

Alzheimer's disease (AD) is one of the most common neurodegenerative disorders. Parenchymal $\beta$ amyloid $(A \beta)$ deposition, which results in neuron death and cognitive decline, is believed to be the main feature of the disease [1]. However, genetic tests, as well as drugs that prevent or even eliminate A $\beta$ deposition, are ineffective, even if they are used in patients with early $\operatorname{AD}[2,3]$. These findings indicate that the etiology of AD may have other possibilities. 
Neuroinflammation plays important roles in several neurological and neurodegenerative diseases such as Parkinson's disease, amyotrophic lateral sclerosis, and AD [4-6]. Studies have shown that chronic neuroinflammation can occur prior to $A \beta$ and tau pathologies in $A D$ [7]. Proinflammatory cytokines, especially interleukin (IL)-1 $\beta$, can drive the neuroinflammatory process, and its level is upregulated in the brain and cerebrospinal fluid (CSF) of AD patients [8]. Moreover, peripheral proinflammatory cytokines can penetrate the blood-brain barrier (BBB), thereby promoting inflammation in the brain [9]. Goehler's study revealed that intraperitoneal injection of IL-1 $\beta$ induces inflammation in the brain, suggesting that this cytokine may serve as a key mediator of crosstalk between the immune system and central nervous system (CNS) [10]. Other studies have reported an association between the plasma IL-1 $\beta$ level and the progression of $A D[11,12]$. However, there is no study on the association between neuroinflammation in CSF and systemic inflammation in Peripheral blood monoculear cells (PBMCs) of AD patients.

Inflammasomes are a group of cytosolic multiprotein complexes that can recognize many stimulatory signals and induced several inflammatory cytokines production [13]. Upon recognition of stimuli from pathogens and damaged tissues, the levels of inflammasome-associated pattern recognition receptors (PRRs), such as the NOD-like receptor (NLR) protein family members NLRP1, NLRP3, and NLRC4, as well as absent-in-melanoma 2 (AIM2) and PYRIN-CARD protein ASC, increased, which resulted in the oligomerization of cytosolic protein complexes [14]. These events activated caspase signaling, leading to the production of IL-1 $\beta$ and IL-18 [13]. There is increasing evidence suggested that by inducing proinflammatory cytokines, especially IL-1 $\beta$, inflammasomes contribute to the neuroinflammation of $A D$ [15]. Gasdermin D (GSDMD), a substrate of caspases-1 and $-4 / 11$, has been identified as an executive molecule in pyroptosis. The cleavage of GSDMD results in N-terminal fragment oligomerization and plasma membrane pore formation, which regulates the secretion of proinflammatory cytokines, including IL-1 $\beta$ [16]. Recent studies have reported that GSDMD-induced pyroptosis is involved in many neurological disorders such as ischemic stroke [17], Parkinson's disease [18], and multiple sclerosis [19]. However, it is unclear whether GSDMD-induced pyroptosis occurred in periphery of AD patients and influences neuroinflammation.

The aim of this study was to investigate the association between systemic inflammation induced by inflammasome-mediated pyroptosis and AD progression.

\section{Materials And Methods}

\section{Participants}

We used the criteria of the National Institute on Aging and the Alzheimer's Association for AD diagnosis [20], and published criteria [21] for amnestic mild cognitive impairment (aMCl) diagnosis. We recruited 33 patients with $\mathrm{AD}$ and 33 patients with aMCl from the Department of Neurology, Affiliated Brain Hospital of Nanjing Medical University, from January 2019 to April 2020. For controls, we recruited 20 agematched control participants with (i) no history of infectious, inflammatory, and autoimmune diseases; (ii) no history of psychiatric and memory disorders; and (iii) no history of prescription and non- 
prescription drug use. All participants or their legal guardians provided informed written consent. This study was approved by the Institutional Review Board of the Affiliated Brain Hospital of Nanjing Medical University.

\section{Collection of PBMCs from blood}

Blood samples were collected in the morning and processed within $4 \mathrm{~h}$. Approximately $3 \mathrm{~mL}$ of anticoagulated whole blood was centrifuged at $3500 \mathrm{rpm}$ for $3 \mathrm{~min}$ at $4^{\circ} \mathrm{C}$, and the plasma was collected and stored at $-80^{\circ} \mathrm{C}$ for the measurement of cytokines. To obtain PBMCs, $2 \mathrm{~mL}$ of whole blood was diluted with phosphate-buffered saline (PBS, 1:1) and transferred to centrifuge tubes containing $3 \mathrm{~mL}$ of Ficoll Paque (GE Healthcare, Uppsala, Sweden). After centrifuging at $400 \times \mathrm{g}$ for $20 \mathrm{~min}$ at room temperature, PBMCs were collected and washed twice in $10 \mathrm{~mL}$ of PBS. Cell pellets were re-suspended in TRIzol or lysis buffer for the isolation of RNA or protein, respectively.

\section{Quantitative polymerase chain reaction (qPCR) analysis}

Total RNA extraction, cDNA synthesis, and qPCR reactions were performed according to the manufacturer's instructions. TRIzol reagent (Invitrogen, CA, USA) was used to extract RNA from PBMCs, and RNA was used for subsequent cDNA synthesis. qPCR was performed using SYBR Premix Ex Taq (Takara Bio, Dalian, China). The following primers were used: II-1b (5'-TGTAGTGGTGGTCGGAGATT-3', forward;5'-ATGATGGCTTATTACAGTGGC-3', reverse), NIrp3 (5'-AGGGCGTTGTCACTCAGGT-3', forward; 5'TCGGAGATTGTGGTTGGG-3', reverse), Gsdmd (5'-AGTGCCAGGGAGGCGTAGAGT-3', forward; 5'TGGGTCTTGCTGGACGAGTG-3', reverse), Caspase 1 (5'-GGAAGAGCAGAAAGCGATAA-3', forward; 5' TTGAAGGACAAACCGAAGG-3', reverse), Caspase4 (5'-TGCCAGGAAAGAGGTAGAAA-3', forward; 5' TCGGAAGGTACAGCAATCA-3', reverse), NIrc4 (5'-GACTAATGCTGGATCAGGTAG-3', forward; 5' TTTGGCGGGAAATCGTGT-3', reverse), Aim2 (5'-TCAGTACCATAACTGGCAAA-3', forward; 5' AGAAATGATGTCGCAAAGC-3', reverse), NIrp1 (5'-AACGTAGAACTCCGAGAAC-3', forward; 5' CGAATCCACAAGCCACCC-3', reverse), and Gapdh (5'-GAAGGTGAAGGTCGGAGTC-3', forward; 5'GAAGATGGTGATGGGATTTC-3', reverse). The relative expression level of each target gene was calculated using a standard curve and normalized against Gapdh expression level.

\section{Immunofluorescence staining}

PBMCs were collected, fixed with $4 \%$ paraformaldehyde (PFA) in 1.5 - $\mathrm{mL}$ centrifuge tubes at $4{ }^{\circ} \mathrm{C}$ for 30 min, washed twice in PBS, blocked with $3 \%$ goat serum, and incubated with primary antibody: rabbit anticleaved N-terminal GSDMD (1:50, Abcam, JHY, UK), that were diluted with PBS containing $3 \%$ serum and $2 \mathrm{mM}$ EDTA at $4{ }^{\circ} \mathrm{C}$ for $12 \mathrm{~h}$, followed by treatment with secondary antibody: Alexa Fluor 555 anti-rabbit (1:500, Invitrogen, CA, USA). For animal experiments, mouse brains were fixed with 4\% PFA, dehydrated with $30 \%$ sucrose, embedded in optimal cutting temperature compound, and sectioned at $25-\mu \mathrm{m}$ thickness. Tissue slices were blocked, incubated with primary antibodies: rabbit anti-IBA1 (1:500, Wako, TKY, JPN), mouse anti-GFAP (1:500, Sigma, CA, USA), or mouse anti-NeuN (1:200, MilliporeSigma, CA, USA), and treated with secondary antibodies: Alexa Fluor 488 anti-rabbit (1:500, Invitrogen, CA, USA), and 
Alexa Fluor 555 anti-mouse (1:500, Invitrogen, CA, USA), followed by staining with 4',6-diamidino-2phenylindole (DAPI) or thioflavin S (Sigma-aldrich, MO, USA).

\section{Collection of cerebrospinal fluid (CSF)}

CSF was collected by lumbar puncture in the morning at the L3/L4 or L4/L5 level. The first 20 drops of CSF were discarded, followed by the collection of approximately $2 \mathrm{~mL}$ of CSF in a polypropylene tube. The samples were centrifuged for $10 \mathrm{~min}$ at $2000 \times \mathrm{g}$ at room temperature to eliminate cells and other insoluble materials, aliquoted, and stored at $-80^{\circ} \mathrm{C}$ until further processing.

\section{Enzyme-linked immunosorbent assay (ELISA)}

Plasma and CSF samples were collected from participants to determinate the protein levels of AD biomarkers and IL-1 $\beta$. The levels of $A D$ biomarkers including $A \beta 1-40, A \beta 1-42, p$-tau-181, t-tau were measured with commercially available ELISA kits (INNOTEST, Fujirebio, Ghent, Belgium) according to the test procedure in manufacturer's protocols. Levels of IL-1 $\beta$ in plasma and CSF were measured with commercial ELISA kits (Jingmei, Yancheng, China). A microplate reader (Antobio, Zhengzhou, China) was used to obtain absorbance readings. For animal experiments, serum and brain tissue were collected from mice and used to detect the protein level of IL-1 $\beta$.

\section{Western blot analysis}

Approximately $1.5 \times 10^{6}$ PBMCs were mixed with $70 \mu \mathrm{L}$ of loading buffer (Yeasen, Shanghai, China) and then boiled in a water bath set to $99{ }^{\circ} \mathrm{C}$ for $10 \mathrm{~min}$. The protein samples and PageRuler Prestained Protein Ladder were loaded onto SDS-polyacrylamide gels, and then blotted onto PVDF membranes. The blots were incubated overnight at $4{ }^{\circ} \mathrm{C}$ with the following primary antibodies: mouse anti-NLRP3 (1:1000, Adipogen Corporation, CA, USA), rabbit anti-caspase-1 (1:1000, Abcam, JHY, UK), rabbit anti-GSDMD (1:200, Novus, Littleton, USA), mouse anti-IL-1 $\beta$ (1:1000, R\&D Systems, Minneapolis, USA), mouse anti- $\beta$ actin (1:4000, Sigma-aldrich, MO, USA). The membranes were then washed three times with Tris-buffered saline and incubated with secondary antibodies: IRDye 800CW goat-anti-mouse/rabbit (1:3000, LI-COR Biosciences, Lincoln, USA). Image $\mathrm{J}$ software was used to analyze the intensities of the bands. In the animal study, mouse spleens were harvested and homogenized 1:10 in homogenization buffer (20 mM Tris-HCl, 150 mM NaCl, 1 mM EGTA, 1 mM EDTA, 2.5 mM sodium pyrophosphate, 1 mM $\beta$-glycerol phosphate, $1 \mathrm{mM}$ sodium vanadate, $1 \%$ Triton $\mathrm{X}-100,1 \mathrm{mg} / \mathrm{mL}$ leupeptin, and protease inhibitor cocktail, $\mathrm{pH}$ 7.5). The concentrations of the protein samples were quantified with the bicinchoninic acid method (Bio-Rad, Hercules, CA, USA) after centrifuging at $12000 \times \mathrm{g}$ for $10 \mathrm{~min}$. The protein samples were then subjected to western blotting analysis as indicated above.

\section{RNA-seq analysis}

For RNA-seq, PBMCs were obtained. RNA isolation, cDNA library construction, and RNA-seq were performed with the BGISEQ-500 system (Beijing Genomic Institution). Clean reads were mapped to the human genome (hg38) by HISAT2. Thereafter, the matched reads were calculated and normalized to FPKM. Fold changes were calculated for all possible comparisons. To select genes with significant 
changes in expression, a 1.5-fold cutoff was used. Gene Ontology biological process (GO-BP) pathway analyses were performed using the $\mathrm{R}$ package. Target genes were filtered using significantly different gene expression $(P<0.05)$. Raw data files and processed files have been deposited in the Gene Expression Omnibus under accession no. GSE265984.

\section{Treatment of mice with lipopolysaccharides}

\section{Animals and treatments}

We used 5-month-old 5×FAD mice, kindly provided by Dr. Ming Xiao (Nanjing Medical University, Jiangsu, China), which carried mutations in both the human amyloid precursor protein (APP695) and presenilin-1 (PSEN1) genes. The APP gene contains three familial AD (FAD) mutations: Swedish (K670N, M671L), Florida (1716V), and London (V717I). The PSEN1 gene contains two FAD mutations: M146L and L286V. Low-dose lipopolysaccharides (LPS; $500 \mu \mathrm{g} / \mathrm{kg}$ ) or PBS control was injected intraperitoneally 48 hours before the mice were sacrificed. In GSDMD inhibitor treatment experiments, disulfiram $(50 \mathrm{mg} / \mathrm{kg})$ or PBS was injected intraperitoneally 4 hours before challenge with LPS. Serum, brain, and spleen tissues were collected for ELISA, western blotting, and immunofluorescence analyses, as described above ( $\mathrm{n}=$ 3 /group). Animal experiments were conducted according to the National Institutes of Health Guidelines for the Care and Use of Laboratory Animals, and all animal procedures were approved by the Ethical Review Committee for Laboratory Animal Welfare of Nanjing Medical University.

\section{Statistical analysis}

Data are shown as the means \pm standard error of the mean (SEM). Statistical analysis was performed with one-way ANOVA followed by Sidak's multiple comparisons test or unpaired t test. The correlative analysis was performed using a linear regression model. Chi-square test was used for the analysis of discrete variable such as sex. All statistical analyses in this study were performed in GraphPad Prism 6.0 software (Graph Pad Software Inc., San Diego, CA, USA). $P$ values are indicated as ${ }^{\star} P<0.05,{ }^{\star \star} P<0.01$, and ${ }^{* \star *} P<0.001$.

\section{Results}

\subsection{Participant characteristics}

A total of 66 patients and 20 healthy control individuals participated in the study. The characteristics of the participants are listed in Table 1. No statistical differences were found in age or sex among the aMCl, $A D$, and control groups. The Mini-Mental State Examination (MMSE) and Montreal Cognitive Assessment (MoCA) scores were significantly different between $\mathrm{aMCl}$ and control groups, $\mathrm{aMCl}$ and $\mathrm{AD}$ groups, and $A D$ and control groups. The expression of $A D$ biomarkers, including CSF $A \beta 1-42, A \beta 1-40$, and $p$-tau-181, differed between patients with $\mathrm{aMCl}$ and patients with $\mathrm{AD}$.

\subsection{Inflammation-related pathways and genes are enriched in PBMCs of patients with AD}


Inflammasomes have been widely confirmed to play important roles in the progression of neuroinflammation. To investigate whether a specific relationship exists between peripheral inflammasome activation and dementia, we first investigated gene transcriptome changes in PBMCs between patients with $A D$ and healthy controls, by performing RNA sequencing analysis. Go biological process analysis indicated that the most significantly changed gene enrichment pathways in patients with $A D$ were immune system process, innate immune response, and inflammatory response, etc (Fig. 1a). Moreover, gene network and gene set enrichment analysis (GSEA) revealed enrichment in the up-regulated genes involved in the inflammatory response pathway in patients with AD (Fig. 1b). These findings agreed with those of the heatmap, which showed the levels of many genes involved in inflammasome-mediated inflammation, such as I/1r1, I/1rap, N/rc4, NIrp6, NIrp9, Aim2, Casp1, and Casp4 were increased in the PBMCs of patients with AD (Fig. 1C). Thus, these genomic analyses implied the upregulation of peripheral inflammasome-mediated inflammation pathways in patients with AD.

\subsection{Activation of inflammasomes and GSDMD in PBMCs from patients with $A D$}

To further confirm the changes in peripheral inflammasomes, we increased the number of clinical samples to analyze the expression of inflammasome associated genes by real-time PCR. As shown in Fig. 1, the gene expression of NLRP3 and AIM2 was higher in patients with aMCl or AD than in the controls (Fig. 2a, b), whereas the expression of NLRP1 and NLRC4 genes did not differ among the groups (Fig. 2c, d). We next detected the gene expression of downstream effector proteins. The results showed that the gene expression of caspase- 1 and IL-1 $\beta$ was higher than that in the control group, but caspase 4 did not differ among the groups (Fig. 2e, f, g). These data were partially consistent with our genomic results. GSDMD has been extensively studied in pyroptosis, and the release of IL-1 $\beta$ is highly dependent on the activation of GSDMD $[22,23]$. Therefore, we detected the GSDMD gene expression in the different groups; to our knowledge, such detection has not been reported previously. Notably, compared with that in the control group, the GSDMD gene expression in the $\mathrm{aMCl}$ and $\mathrm{AD}$ groups was higher, as expected (Fig. 2h).

\subsection{Inflammasome-induced pyroptosis drives systemic inflammation in patients with $A D$}

To confirm the changes in inflammasomes at the protein level in PBMCs during AD, we then investigated the protein expression of the main components of the inflammasome pathway by western blotting. The expression of NLRP3, cleaved caspase-1, and mature IL- $1 \beta$ relative to that of $\beta$-actin in PBMC lysates from patients with $\mathrm{aMCl}$ was greater than that in the controls, and was significantly greater in patients with AD than the controls (Fig. 3a, b). More importantly, the cleavage of GSDMD markedly increased in $\mathrm{aMCl}$ and $\mathrm{AD}$, thus indicating the exacerbation of pyroptosis induced by GSDMD. Next, we visualized the proportion of pyroptosis in PBMCs through immunofluorescence, the numbers of GSDMD N-terminal positive cells in PBMCs from patients with $A D$ were markedly greater than those in the controls (Fig. 3c, 


\subsection{Plasma levels of IL-1 $\beta$ are positively correlated with AD progression}

Because plasma samples are relatively easy to obtain, they are often used as samples for analyses, thus offering the prospect of readily measurable biomarkers for many diseases. In this study, we detected the IL-1 $\beta$ concentrations in the plasma from patients with aMCl, patients with $A D$, and controls by ELISA. The levels of plasma IL-1 $\beta$ in the patients with $\mathrm{aMCl}$ or $A D$ were significantly higher than those in the controls (Fig. 4a). A $A 1-42$ in the CSF is an important biomarker of $A D$, and decreased $A \beta 1-42$ levels in the CSF indicate the accumulation of $A \beta 1-42$ in the brain parenchyma [24]. Therefore, we detected whether the plasma levels of IL-1 $\beta$ correlated with the CSF levels of $A \beta 1-42$. The results showed a negative correlation between the two in patients with $\mathrm{aMCl}$ or $\mathrm{AD}$ (Fig. 4b, e). MMSE and MoCA are currently the most commonly used scales in cognitive function evaluation in clinical settings; lower scores imply more severe cognitive impairment. We also examined the correlation between plasma levels of IL-1 $\beta$ and the MMSE and MoCA scores in patients. The levels of IL-1 $\beta$ and the MMSE score were highly negatively correlated in both patients with $\mathrm{aMCl}$ and those with $\mathrm{AD}$ (Fig. 4c, d). However, the negative correlation between IL-1 $\beta$ levels and the MoCA score was found only in patients with AD (Fig. $4 f, g$ ).

\subsection{IL-1 $\beta$ level in CSF is positively correlated with AD progression}

The IL-1 $1 \beta$ level in CSF from $A D$ and aMCI patients was measured to determine the severity of inflammation in the brain. We examined the correlation between the IL-1 $\beta$ level and clinical characteristics such as biomarkers in CSF and psychological evaluation. We found a weak negative correlation between the levels of IL-1 $\beta$ and $A \beta 1-42$ in CSF from $A D$ and aMCl patients (Fig. $5 a, d$ ), and a significant correlation between the IL-1 $\beta$ level and MMSE and MoCA scores (Fig. 5b, c, e, f), indicating that IL-1 $\beta$, which associates with the progression of $A D$, may be as a potential biomarker of neuroinflammation in $A D$.

\subsection{Positive correlation between IL-1 $\beta$ levels in the plasma and $\mathrm{CSF}$ in patients with aMCI or $\mathrm{AD}$}

We next detected whether the IL-1 $\beta$ levels in CSF correlated with the plasma IL-1 $\beta$ levels. The levels of IL$1 \beta$ in the CSF from patients with aMCl or AD were highly positively correlated with the plasma IL-1 $1 \beta$ levels (Fig. 6). This result implied that the level of peripheral inflammation is strongly associated with the level of central inflammation in patients with AD. 


\subsection{Peripheral inflammation aggravates neuroinflammation in 5×FAD mice}

The development of neuroinflammation in neurodegenerative disorders is not only triggered by damage signals within the brain but also promoted by proinflammatory cytokines released from peripheral. To verify the influence of peripheral pyroptosis activation on neuroinflammation in an animal AD model, we intraperitoneally injected low-dose LPS into 5xFAD mice to induce the peripheral inflammation activation without crossing the blood-brain barrier, as previously reported [25]. The level of IL-1 $\beta$ in the serum in $5 \times$ FAD mice treated with LPS was markedly greater than that in mice treated with PBS (Fig. 7a). Meanwhile, the activation of GSDMD in the spleen was enhanced by the administration of LPS (Fig. 7b, c). As expected, the level of IL-1 $\beta$ in the brain also increased, although not significantly, as it did in the serum, thus suggesting an influence of peripheral GSDMD activation on brain microenvironments (Fig. 7a). Next, we explored the changes in AD pathological features after the administration of LPS in $5 \times F A D$ mice. Although, peripheral GSDMD and IL-1 $\beta$ did not affect the deposition of A $\beta$ plaques and the survival of neurons around the hippocampus, they enhanced the activation of microglia in the same area (Fig. 7d, e), probably because microglia, as CNS innate immune cells, are more sensitive to microenvironmental changes and can respond quickly. Notably, the injection of the GSDMD inhibitor disulfiram not only inhibited the activation of peripheral inflammation induced by LPS but also decreased the activation of microglia in the brain. Unexpectedly, disulfiram also appeared to decrease the peripheral and brain inflammation in 5×FAD mice without LPS stimulation.

\section{Discussion}

Many studies have indicated that systemic inflammation plays important roles in neurodegenerative diseases including AD [26]. The present study provides convincing evidence that inflammasomes and downstream pyroptosis are both activated in the PBMCs of patients with aMCl or AD. RNA-seq analysis revealed that gene enrichment pathways associated with the inflammatory response are upregulated in patients with AD. The mRNA levels and the protein activation of inflammasome related components in the PBMCs of patients with $\mathrm{aMCl}$ or $\mathrm{AD}$ were greater than those in the controls. In addition, the levels of IL-1 $\beta$ in plasma were significantly elevated in patients with $\mathrm{AMCl}$ or $\mathrm{AD}$, and were negatively correlated with the CSF A $1-42$ levels, and MMSE and MoCA scores. We also observed that the IL-1 $\beta$ levels in CSF were negatively correlated with the CSF A $\beta 1-42$ levels, and MMSE and MoCA scores. Finally, a positive correlation between CSF IL-1 $\beta$ levels and plasma IL-1 $\beta$ levels was confirmed. These findings suggested canonical inflammasome and pyroptosis activation in the PBMCs of patients with aMCI or AD. IL-1 $\beta$ in the periphery correlated with IL-1 $\beta$ within the CNS, was also associated with the process of AD. We also used an animal model to verify the effects of peripheral pyroptosis in AD. LPS enhanced inflammation and pyroptosis in the periphery, whereas targeted inhibition of GSDMD decreased neuroinflammation in $A D$ mice. 
In the CNS, neuroinflammation are known to play important roles in the progression of AD [6]. Recent studies have shown that not only CSF, but plasma IL-1 $\beta$ concentrations are also significantly higher in $A D$ and $\mathrm{MCl}$ than in healthy control $[27,28]$. Peripheral inflammatory cytokines can penetrate the blood-brain barrier and increase brain inflammation, thereby accelerating the pathological process of AD [9]. Consistently, in our study the levels of IL-1 $\beta$ in plasma were significantly higher in patients with $A D$ and aMCI than in controls, and showed a positive correlation with the CSF IL-1 $\beta$. This finding indicated that peripheral inflammation is closely associated with CNS inflammation. Inflammasome induced systemic inflammation plays an important role in nervous system diseases [29-31]. The canonical

NLRP3/caspase-1 inflammasome is the most studied inflammasome in AD. Studies have shown that in amyloid-plaque containing mice and patients with AD, NLRP3 related gene and protein expression is elevated [32, 33]. Moreover, ASC or NLRP3 knockout decreases the amyloid plaque and Tau pathology in $A D$ transgenic mice $[34,35]$. In our study, we report the first detection of inflammasome related genes and proteins in PBMCs from patients with $\mathrm{aMCl}$ or $\mathrm{AD}$, and control participants. The inflammasome pathway was significantly activated in the PBMCs of patients with aMCl or AD.

The downstream molecular events of inflammasome activation in pyroptosis was unclear until GSDMD was identified [16]. Inflammasome-induced cleavage of GSDMD N-terminus leading to plasma membrane pore formation, eventually inducing pyroptosis and release IL-1 $\beta[22,23]$. GSDMD can be activated by both canonical and non-canonical inflammasomes, and thus has broad effects on pyroptosis induced inflammation. We hope that this study will provide information on the association between peripheral GSDMD-induced inflammation and $A D$ that will be useful for future work. Recent studies have shown that disulfiram specifically inhibits the pyroptosis induced by $\operatorname{GSDMD}[19,36]$. Our AD animal model showed that administration of disulfiram inhibits peripheral inflammation and alleviates microglial activation during $A D$. After validation of these target proteins as biomarkers for diagnosis or assessment of disease progression in $A D$, the next step will be to target the biomarkers for potential new and improved treatments for the growing number of people with $A D$ worldwide.

\section{Conclusions}

In summary, our results provide evidence that the NLRP3/caspase-1/GSDMD pathway is activated in the periphery of patients with $\mathrm{aMCl}$ and patients with $\mathrm{AD}$. Peripheral inflammasome-induced pyroptosis increases IL-1 $\beta$ and exacerbates the pathological process of AD.

\section{Abbreviations}

AD: Alzheimer's disease; GSDMD: gasdermin D; aMCl: amnestic mild cognitive impairment; MMSE: Minimental State Examination; MoCA: Montreal Cognitive Assessment; PBMCs: peripheral blood mononuclear cells; CSF: Cerebrospinal fluid; LPS: lipopolysaccharides; A $\beta$ : $\beta$-amyloid; BBB: blood-brain barrier; CNS: central nervous system; PRRs: pattern recognition receptors; NLR: NOD-like receptor; ASC: apoptosisassociated speck-like protein containing a CARD; AIM2: absent-in-melanoma 2; PFA: paraformaldehyde; 
ELISA: Enzyme-linked immunosorbent assay; FAD: familial AD; SEM : standard error of the mean; GSEA: gene set enrichment analysis

\section{Declarations}

\section{Acknowledgments}

We appreciate for all the participant in this research, and thanks to the Affiliated Brain Hospital of Nanjing Medical University support this work.

\section{Funding}

This research was supported by the China Postdoctoral Science Foundation (No. 2020M681666).

\section{Availability of data and materials}

The datasets and materials supporting the conclusions of this article are included within the article.

\section{Author contributions}

R.W.J., L.S., F.Y., and M.Z.X performed the experiments, analyzed the data, prepared the manuscript, and prepared the figures; X.H. and X.M. provided mentorship in key techniques and supervised the project; R.W.J., L.S., and S.J.P. designed the study and wrote the manuscript.

\section{Ethics approval and consent to participate}

We obtained informed consent from all participants, and was approved by the ethics committee affiliated Brain Hospital of Nanjing Medical University (Ethical approval number: 2019-KY051-01).

\section{Consent for publication}

Not applicable.

\section{Disclosure statement}

The authors declare no competing financial interests.

\section{References}

1. Hardy, J. and D.J. Selkoe, The amyloid hypothesis of Alzheimer's disease: progress and problems on the road to therapeutics. Science, 2002. 297(5580): p. 353-6.

2. Elmaleh, D.R., et al., Developing Effective Alzheimer's Disease Therapies: Clinical Experience and Future Directions. J Alzheimers Dis, 2019. 71(3): p. 715-732. 
3. Parasrampuria, D.A., L.Z. Benet, and A. Sharma, Why Drugs Fail in Late Stages of Development: Case Study Analyses from the Last Decade and Recommendations. Aaps j, 2018. 20(3): p. 46.

4. Hirsch, E.C. and S. Hunot, Neuroinflammation in Parkinson's disease: a target for neuroprotection? Lancet Neurol, 2009. 8(4): p. 382-97.

5. McCauley, M.E. and R.H. Baloh, Inflammation in ALS/FTD pathogenesis. Acta Neuropathol, 2019. 137(5): p. 715-730.

6. Leng, F. and P. Edison, Neuroinflammation and microglial activation in Alzheimer disease: where do we go from here? Nat Rev Neurol, 2021. 17(3): p. 157-172.

7. Akiyama, H., et al., Inflammation and Alzheimer's disease. Neurobiol Aging, 2000. 21(3): p. 383-421.

8. Hesse, R., et al., Decreased IL-8 levels in CSF and serum of AD patients and negative correlation of MMSE and IL-1ß. BMC Neurol, 2016. 16(1): p. 185.

9. Zenaro, E., G. Piacentino, and G. Constantin, The blood-brain barrier in Alzheimer's disease. Neurobiol Dis, 2017. 107: p. 41-56.

10. Goehler, L.E., et al., Interleukin-1 induces c-Fos immunoreactivity in primary afferent neurons of the vagus nerve. Brain Res, 1998. 804(2): p. 306-10.

11. Scarabino, D., et al., Relationship between proinflammatory cytokines (II-1beta, II-18) and leukocyte telomere length in mild cognitive impairment and Alzheimer's disease. Exp Gerontol, 2020. 136: p. 110945.

12. King, E., et al., Peripheral inflammation in prodromal Alzheimer's and Lewy body dementias. J Neurol Neurosurg Psychiatry, 2018. 89(4): p. 339-345.

13. Broz, P. and V.M. Dixit, Inflammasomes: mechanism of assembly, regulation and signalling. Nat Rev Immunol, 2016. 16(7): p. 407-20.

14. Man, S.M. and T.D. Kanneganti, Regulation of inflammasome activation. Immunol Rev, 2015. 265(1): p. 6-21.

15. White, C.S., et al., Inflammasomes as therapeutic targets for Alzheimer's disease. Brain Pathol, 2017. 27(2): p. 223-234.

16. Shi, J., et al., Cleavage of GSDMD by inflammatory caspases determines pyroptotic cell death. Nature, 2015. 526(7575): p. 660-5.

17. Wang, K., et al., Ablation of GSDMD Improves Outcome of Ischemic Stroke Through Blocking Canonical and Non-canonical Inflammasomes Dependent Pyroptosis in Microglia. Front Neurol, 2020. 11: p. 577927.

18. Rui, W., et al., Baicalein Attenuates Neuroinflammation by Inhibiting NLRP3/caspase-1/GSDMD Pathway in MPTP Induced Mice Model of Parkinson's Disease. Int J Neuropsychopharmacol, 2020. 23(11): p. 762-73.

19. Li, S., et al., Gasdermin D in peripheral myeloid cells drives neuroinflammation in experimental autoimmune encephalomyelitis. J Exp Med, 2019. 216(11): p. 2562-2581. 
20. McKhann, G.M., et al., The diagnosis of dementia due to Alzheimer's disease: recommendations from the National Institute on Aging-Alzheimer's Association workgroups on diagnostic guidelines for Alzheimer's disease. Alzheimers Dement, 2011. 7(3): p. 263-9.

21. Albert, M.S., et al., The diagnosis of mild cognitive impairment due to Alzheimer's disease: recommendations from the National Institute on Aging-Alzheimer's Association workgroups on diagnostic guidelines for Alzheimer's disease. Alzheimers Dement, 2011. 7(3): p. 270-9.

22. Xia, S., et al., Gasdermin D pore structure reveals preferential release of mature interleukin-1. Nature, 2021.

23. He, W.T., et al., Gasdermin D is an executor of pyroptosis and required for interleukin-1 $\beta$ secretion. Cell Res, 2015. 25(12): p. 1285-98.

24. Olsson, B., et al., CSF and blood biomarkers for the diagnosis of Alzheimer's disease: a systematic review and meta-analysis. Lancet Neurol, 2016. 15(7): p. 673-684.

25. Wendeln, A.C., et al., Innate immune memory in the brain shapes neurological disease hallmarks. Nature, 2018. 556(7701): p. 332-338.

26. Holmes, C., Review: systemic inflammation and Alzheimer's disease. Neuropathol Appl Neurobiol, 2013. 39(1): p. 51-68.

27. D'Anna, L., et al., Serum Interleukin-10 Levels Correlate with Cerebrospinal Fluid Amyloid Beta Deposition in Alzheimer Disease Patients. Neurodegener Dis, 2017. 17(4-5): p. 227-234.

28. Eriksdotter, M., et al., Cerebrospinal fluid from Alzheimer patients affects cell-mediated nerve growth factor production and cell survival in vitro. Exp Cell Res, 2018. 371(1): p. 175-184.

29. Fan, Z., et al., Systemic activation of NLRP3 inflammasome and plasma a-synuclein levels are correlated with motor severity and progression in Parkinson's disease. J Neuroinflammation, 2020. 17(1): p. 11.

30. White, A.J., et al., The Peripheral Inflammatory Response to Alpha-Synuclein and Endotoxin in Parkinson's Disease. Front Neurol, 2018. 9: p. 946.

31. Martin, B.N., et al., T cell-intrinsic ASC critically promotes $T(H) 17$-mediated experimental autoimmune encephalomyelitis. Nat Immunol, 2016. 17(5): p. 583-92.

32. Saresella, M., et al., The NLRP3 and NLRP1 inflammasomes are activated in Alzheimer's disease. Mol Neurodegener, 2016. 11: p. 23.

33. Heneka, M.T., et al., NLRP3 is activated in Alzheimer's disease and contributes to pathology in APP/PS1 mice. Nature, 2013. 493(7434): p. 674-8.

34. Ising, C., et al., NLRP3 inflammasome activation drives tau pathology. Nature, 2019. 575(7784): p. 669-673.

35. Venegas, C., et al., Microglia-derived ASC specks cross-seed amyloid- $\beta$ in Alzheimer's disease. Nature, 2017. 552(7685): p. 355-361.

36. Hu, J.J., et al., FDA-approved disulfiram inhibits pyroptosis by blocking gasdermin D pore formation. Nat Immunol, 2020. 21(7): p. 736-745. 


\section{Tables}

Due to technical limitations, table 1 is only available as a download in the Supplemental Files section.

\section{Figures}

a

\section{GO Pathway}

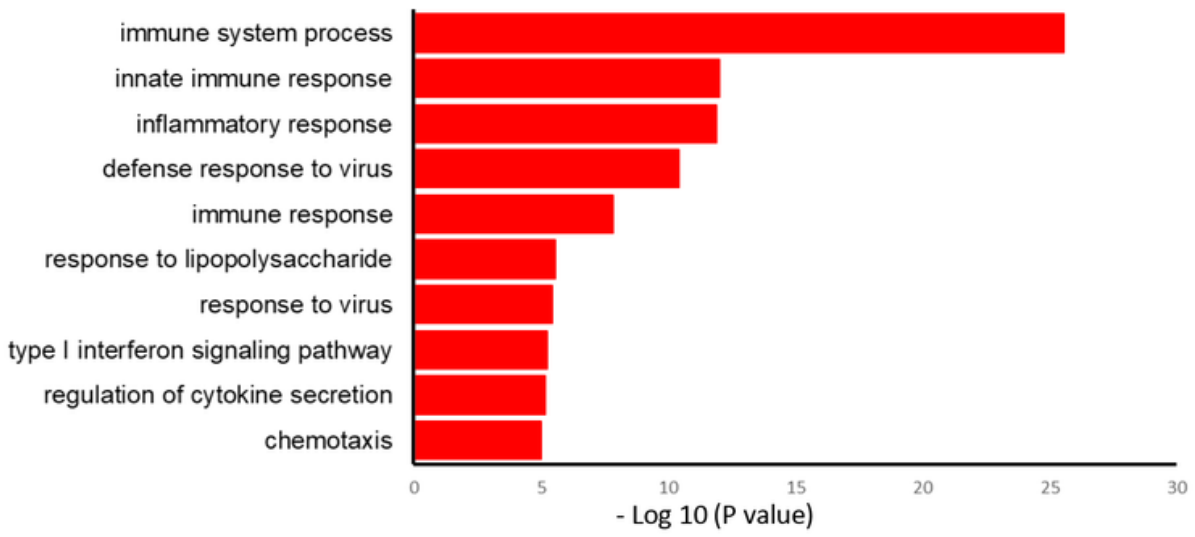

b

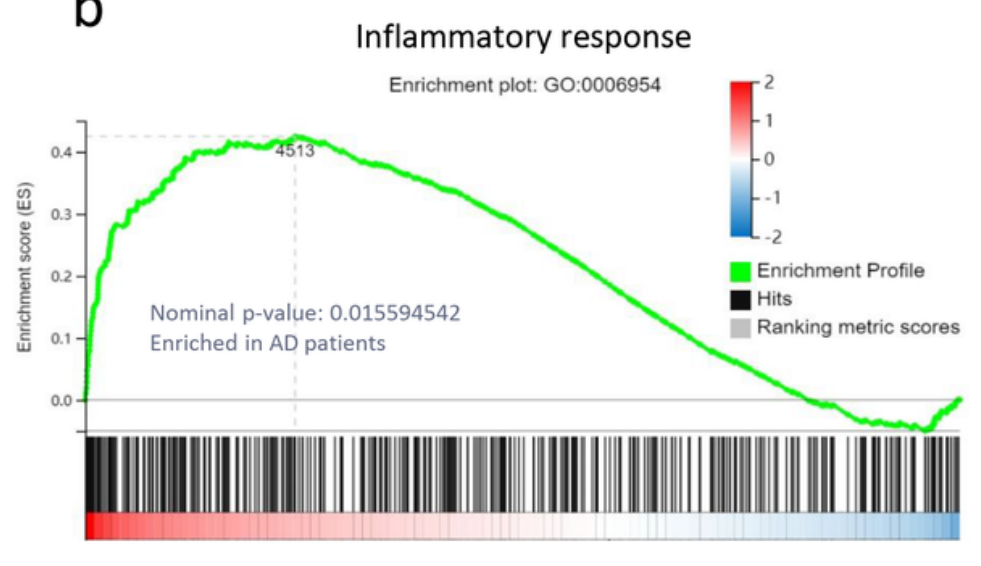

$\mathrm{C} \quad \mathrm{HC}$

$A D$

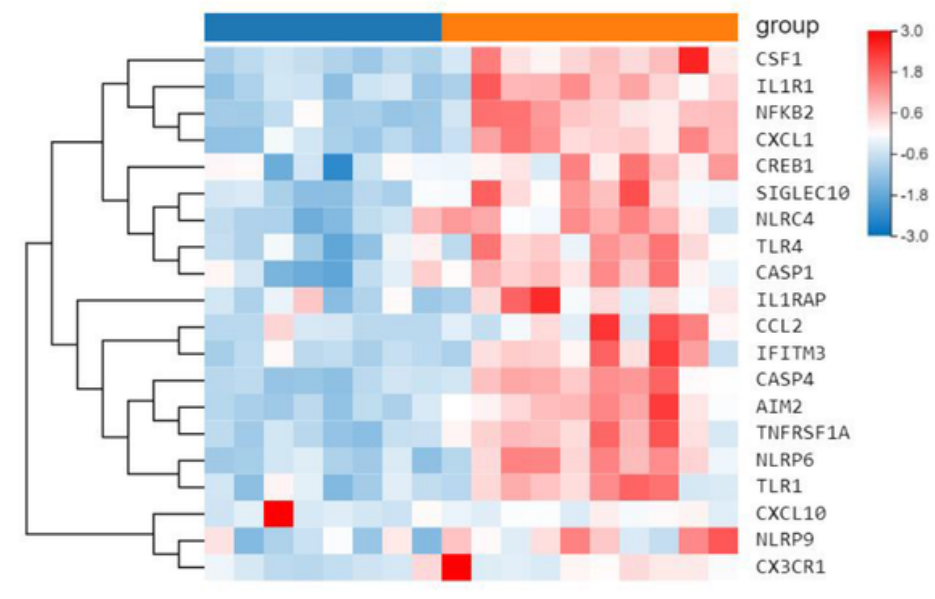

\section{Figure 1}

RNA-seq analysis of PBMCs from patients with AD and healthy controls. (a) GO-BP analysis showing the most significantly enriched signaling pathways in PBMCs. (b) GSEA of the genes associated with "inflammatory response" in PBMCs, on the basis of the Gene Ontology Biological Process Database. Nominal $P<0.05$. (c) The heatmap of genes with adjusted $P$ value $<0.05$, false discovery rate $<0.05$, and log2 fold-change $>1.5$ from RNA-seq of PBMCs. 
a
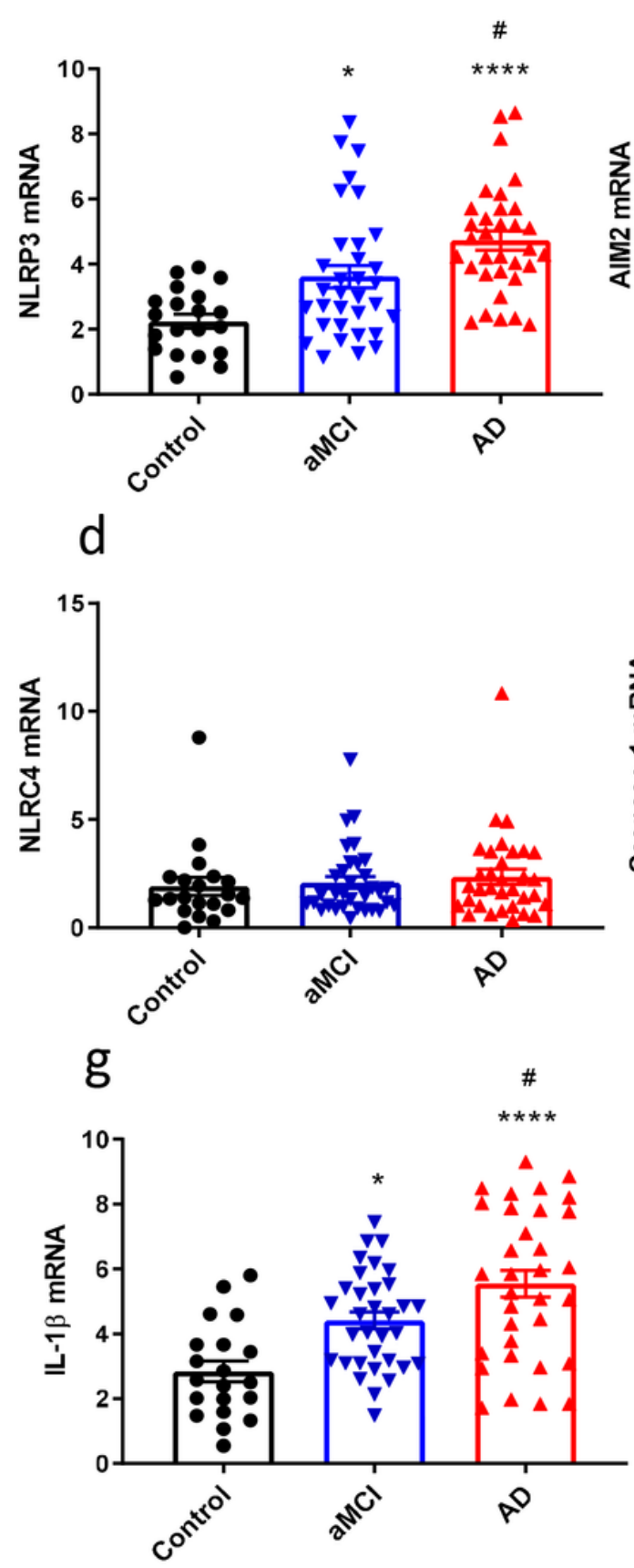

b

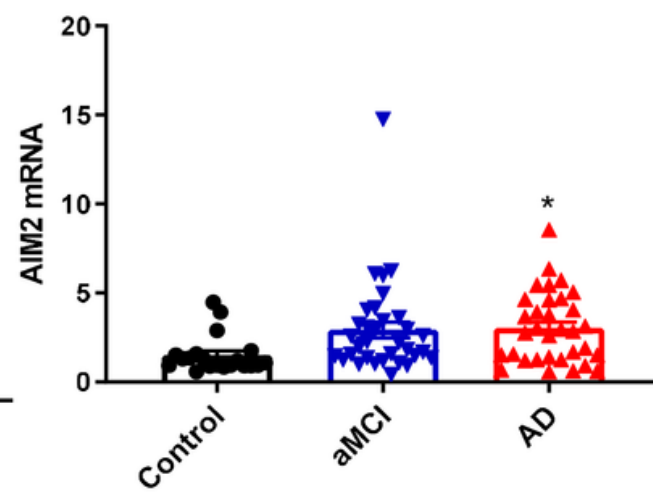

e
C
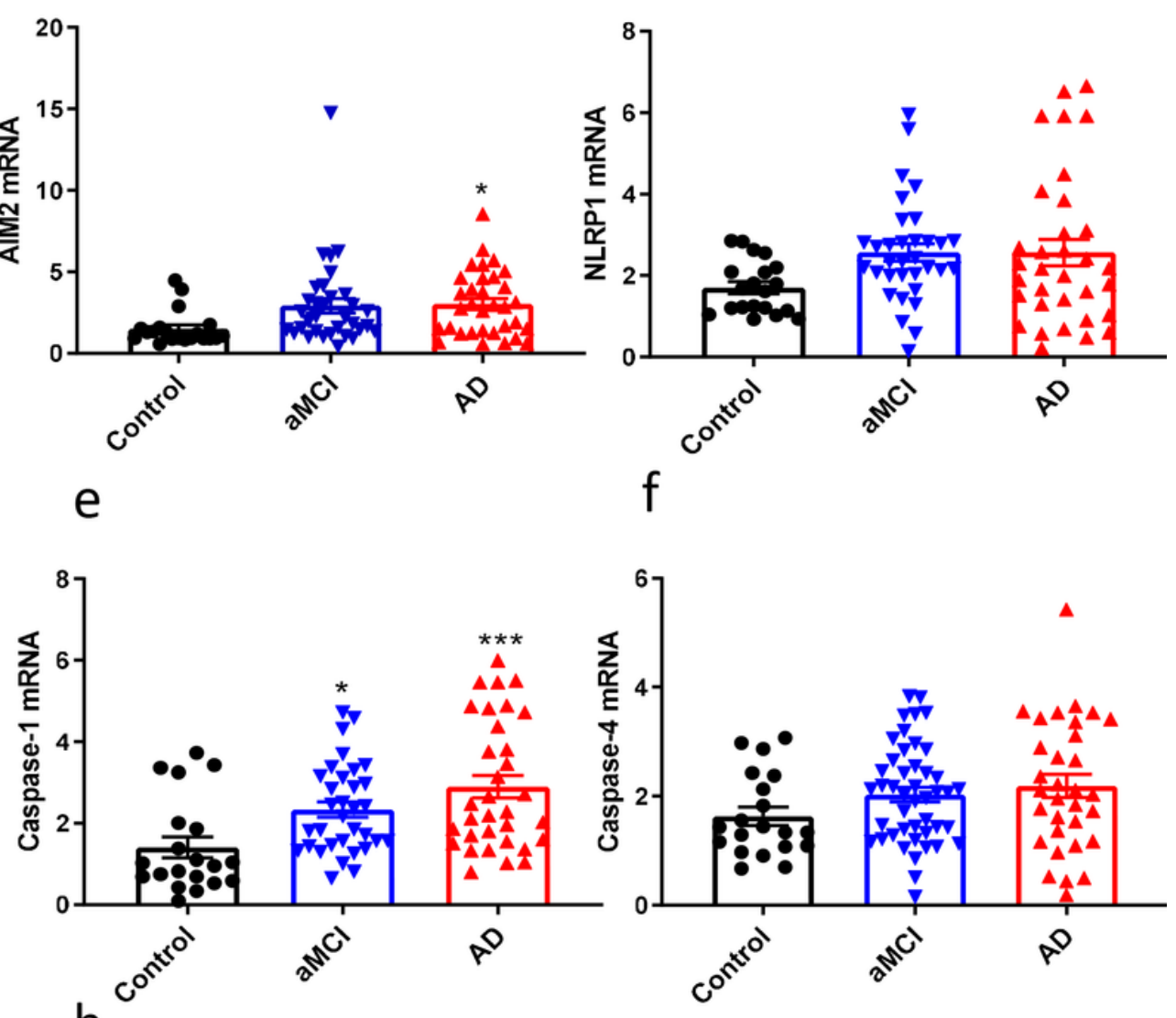

$\mathrm{h}$

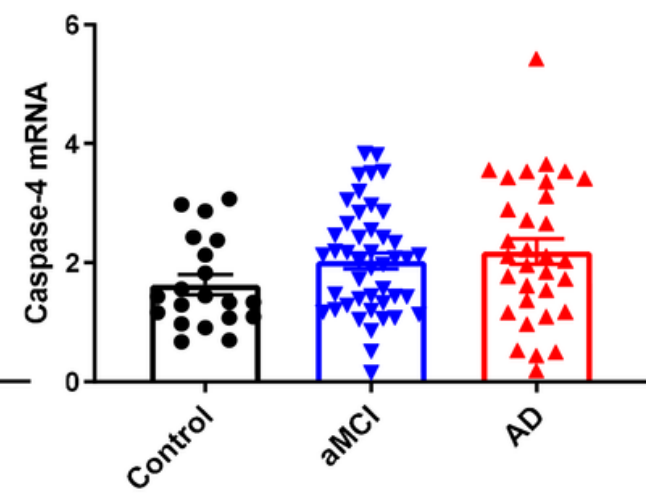

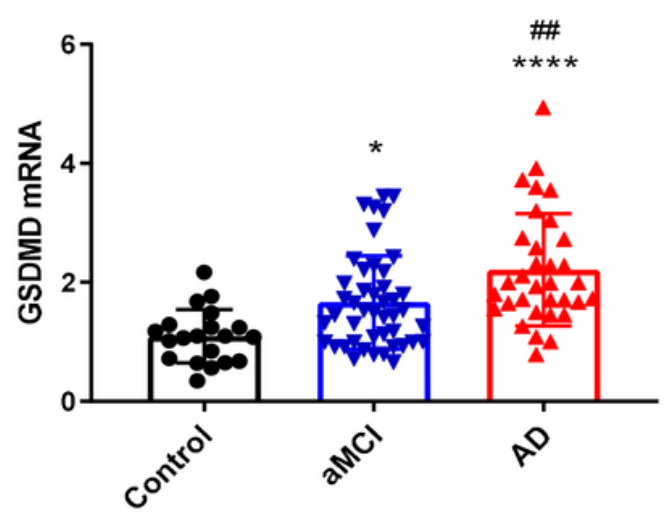

Figure 2

Expression of inflammasome-related genes in the PBMCs of patients with $A D$ and healthy controls. (a-h) The mRNA expression levels of the indicated genes relative to GAPDH were determined by RT-qPCR analysis. ${ }^{*} \mathrm{P}<0.05,{ }^{\star} * \star \mathrm{P}<0.001,{ }^{* \star \star *} \mathrm{P}<0.001$ versus control group, $\# \mathrm{P}<0.05, \# \# \mathrm{P}<0.01$ versus aMCl group. $n=20$ for control, $n=33$ for $a M C l, n=33$ for $A D$. Data are expressed as means $\pm S E M(n=5)$, Oneway ANOVA. 


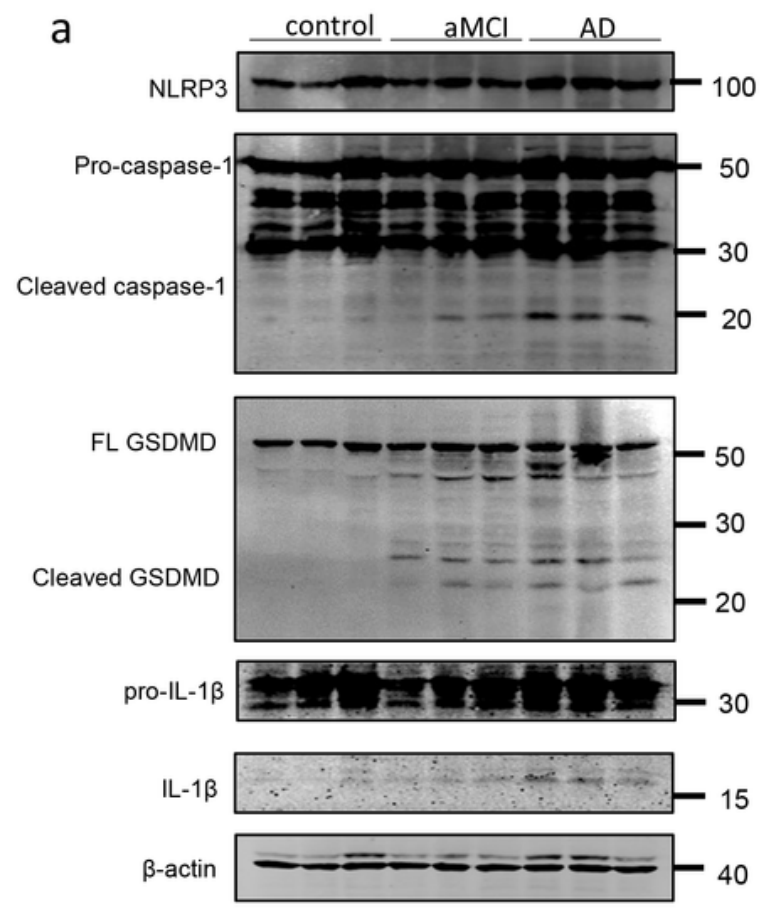

b
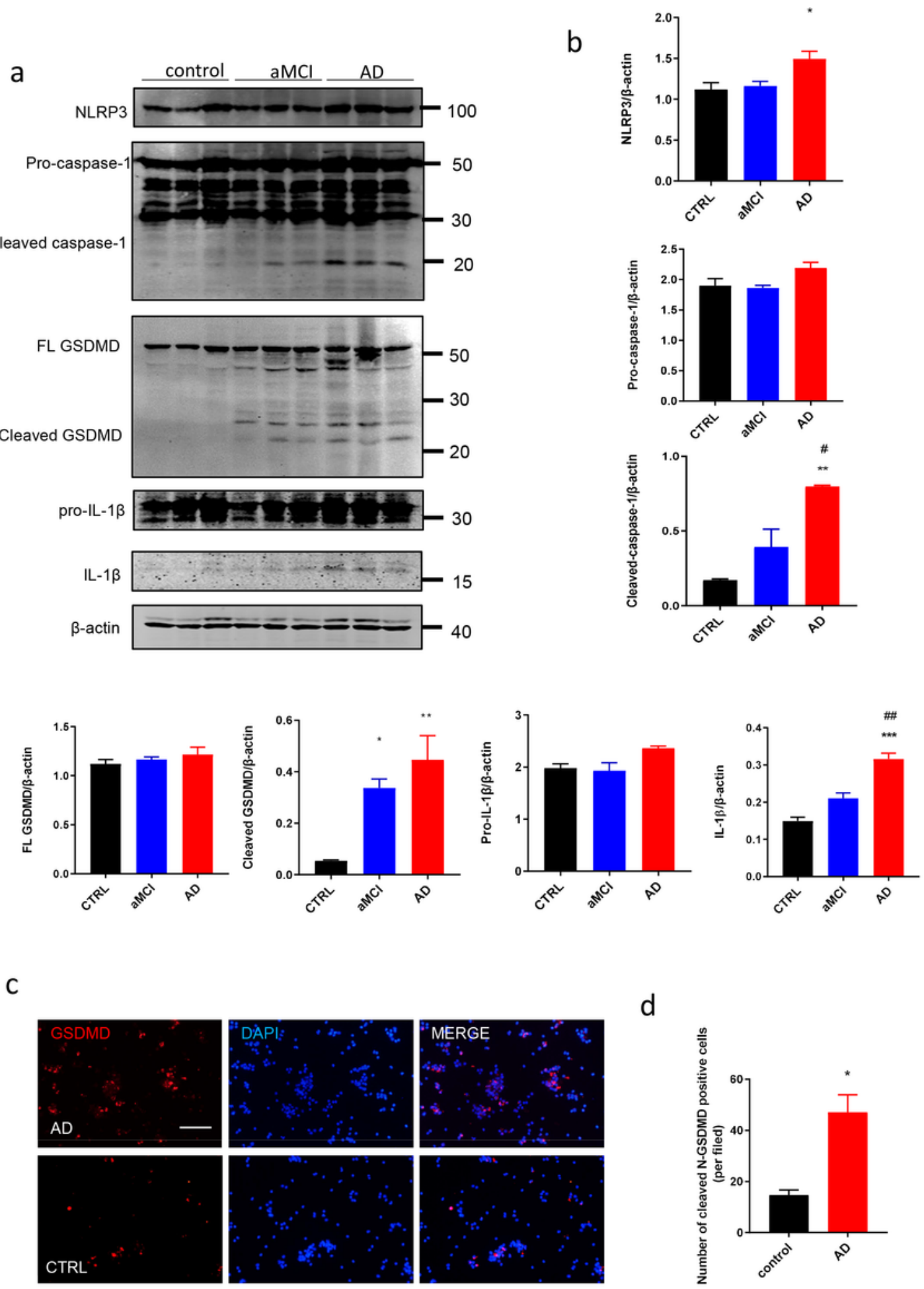

d
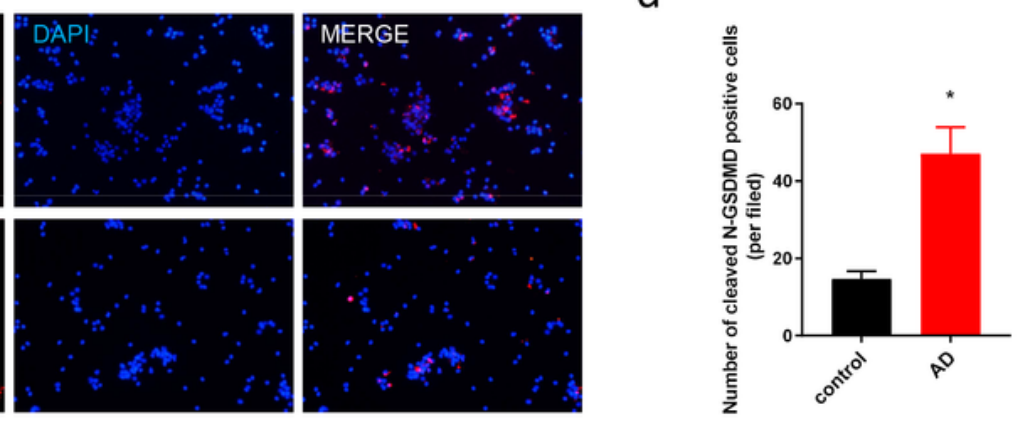

\section{Figure 3}

Activation of inflammasome and pyroptosis proteins in the PBMCs of patients with $A D$ and healthy controls. (a) Expression of NLRP3 protein, cleaved caspase-1, and GSDMD protein, as well as mature IL$1 \beta$ protein, was determined by western blotting in PBMCs from three representative patients with $A D$, three representative patients with aMCl, and three control participants. (b) Statistics of quantified relative protein expression in a. (c) Immunofluorescence analysis of GSDMD N-terminus using PBMCs from a 
representative AD patient and control. (d) Quantified numbers of positive cells in $c$. ${ }^{*} P<0.05, * * P<0.01$, $\star \star \star P<0.001$ versus control group, $\# \mathrm{P}<0.05, \# \# \mathrm{P}<0.01$ versus aMCl group. Data are expressed as means \pm SEM $(n=3)$. One-way ANOVA for $b$. Unpaired t-test for $d$.

a
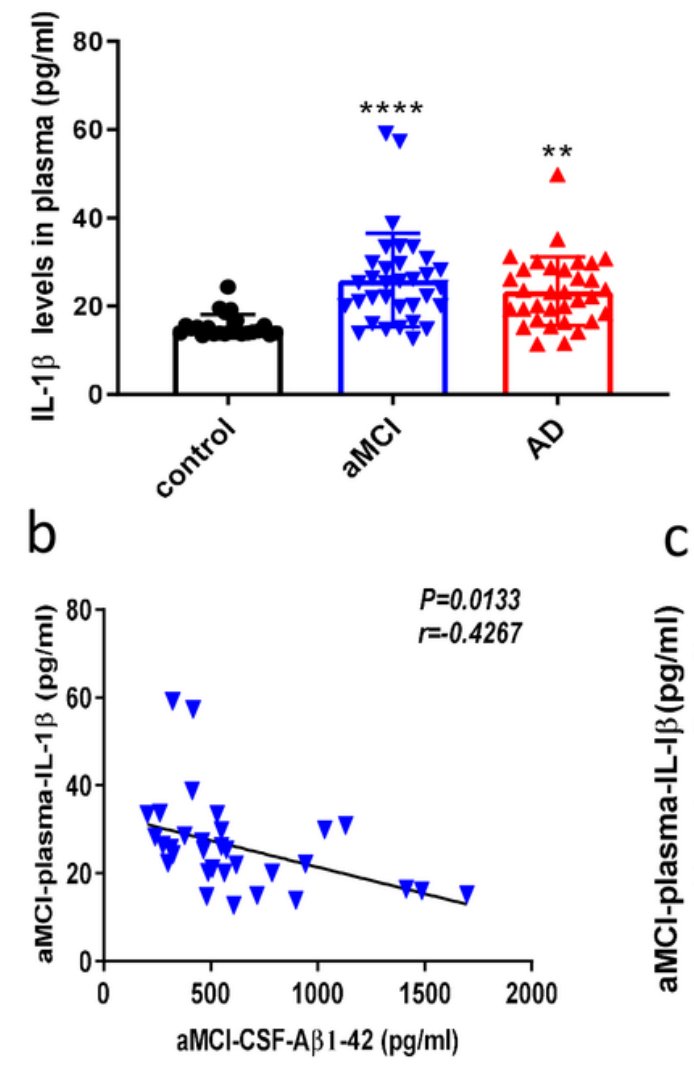

C
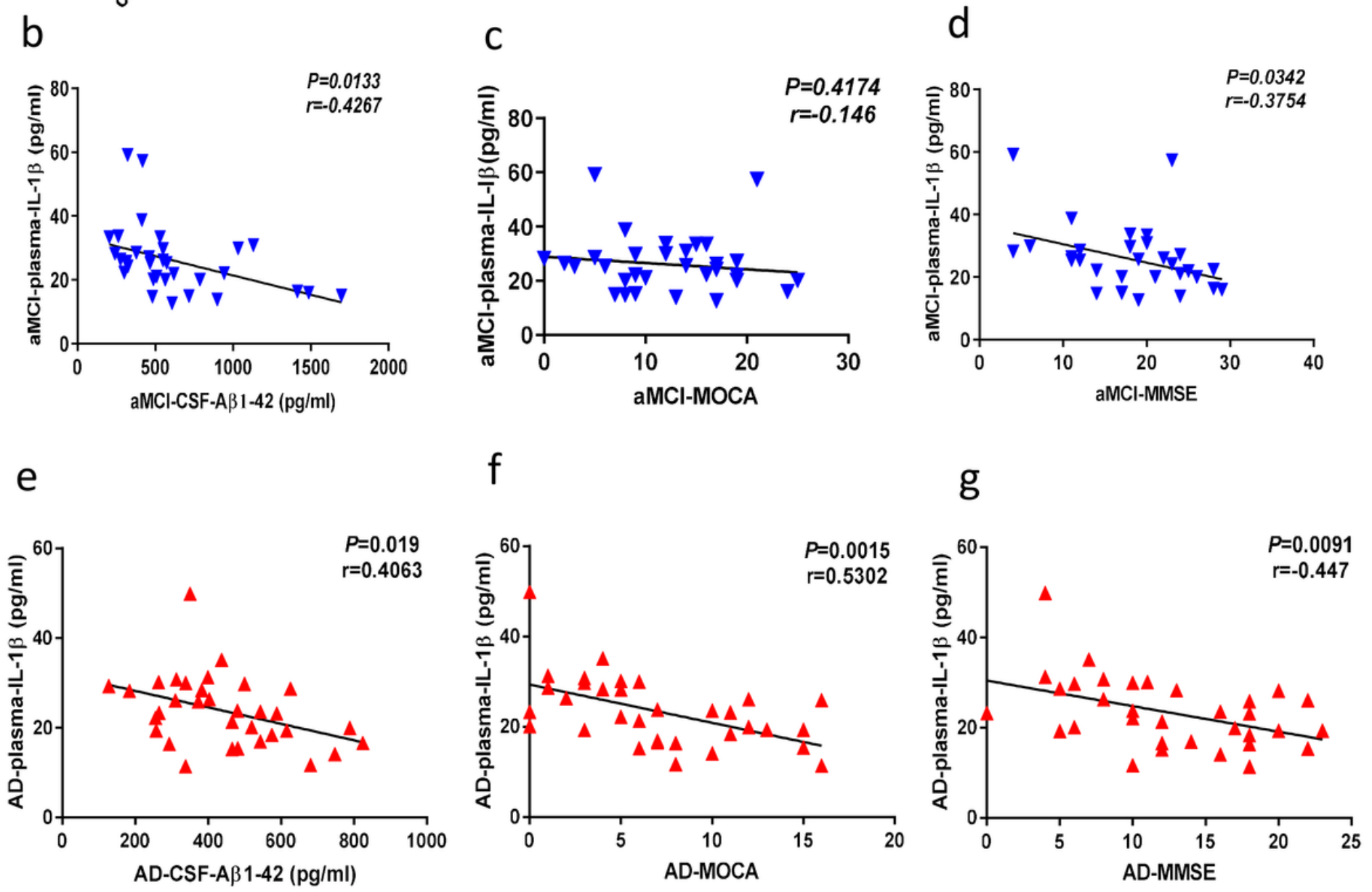

\section{Figure 4}

Plasma levels of IL-1 $\beta$ in patients with $\mathrm{aMCl}$, patients with $\mathrm{AD}$, and healthy controls, and their correlation with clinical characteristics. (a) The expression levels of IL-1 $\beta$ protein measured by ELISA in plasma from patients with $A D$, patients with $\mathrm{aMCl}$, and healthy control participants. (b) Correlation of the IL-1 $1 \beta$ levels in 
plasma from patients with $\mathrm{aMCl}$ and the CSF AB1-42, and MoCA and MMSE scores. (c) Correlation of the IL-1 $\beta$ levels in plasma from patients with AD with the CSF A $1-42$, and MoCA and MMSE scores. **P $<0.01, * \star * * P<0.001$. $n=20$ for control, $n=33$ for $a M C l, n=33$ for AD. Data are expressed as means \pm SEM. One-way ANOVA for a. The correlation was established by calculation of correlation coefficients.

a

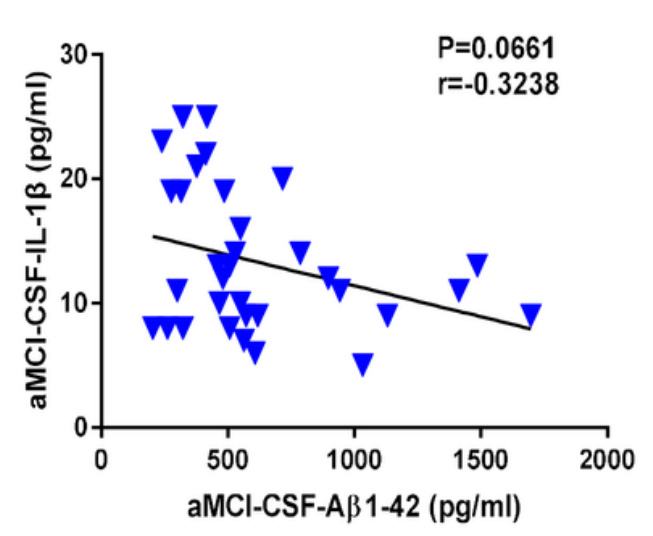

d

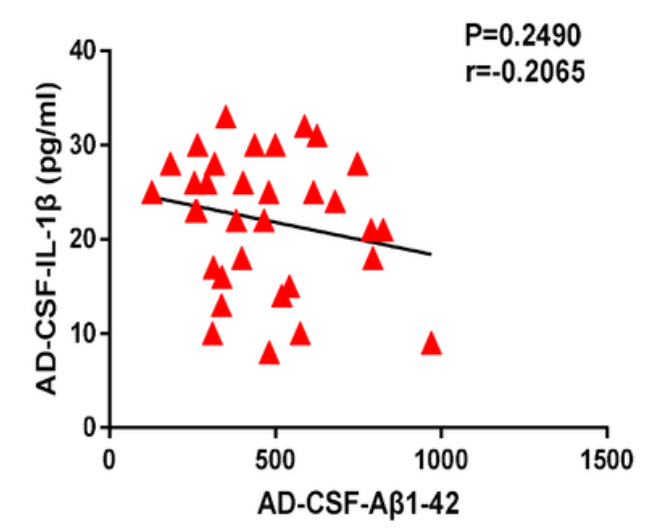

b

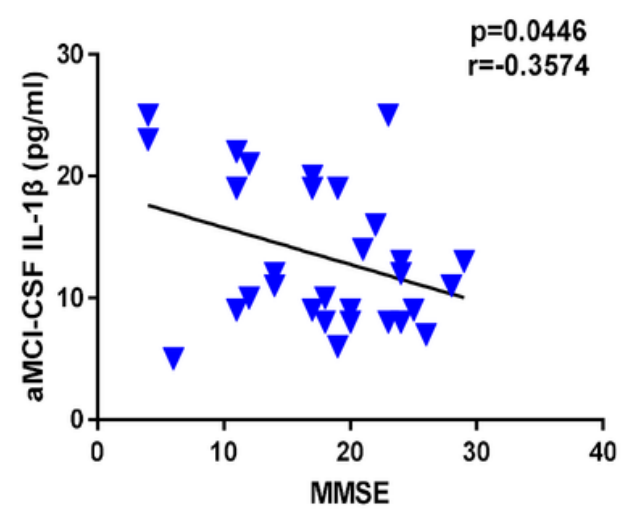

e

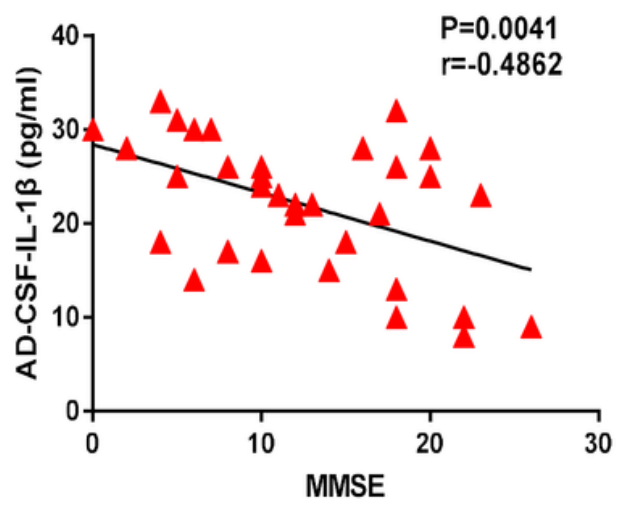

C

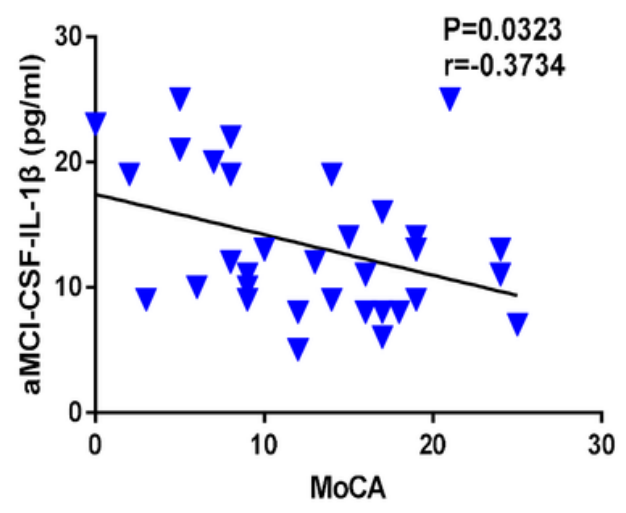

$f$

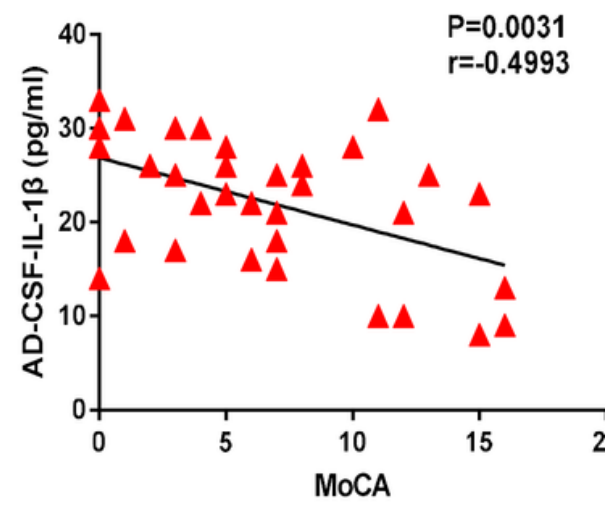

Figure 5

CSF levels of IL-1 $\beta$ in patients with $\mathrm{aMCl}$ and patients with $A D$, and their correlation with clinical characteristics. (a) Correlation of the IL-1 $\beta$ levels in CSF from patients with aMCI with the CSF A $1-42$, and MoCA and MMSE scores. (b) Correlation of the IL-1 $\beta$ levels in CSF from patients with AD with the CSF A $1-42$, and MoCA and MMSE scores. $n=33$ for $a M C l, n=33$ for AD. The correlation was established by calculation of correlation coefficients. 


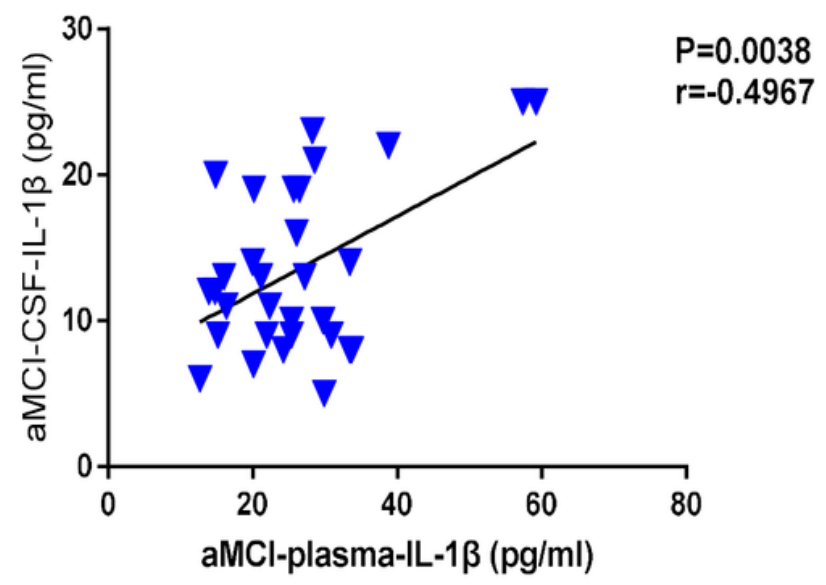

$\mathrm{aMCl}$

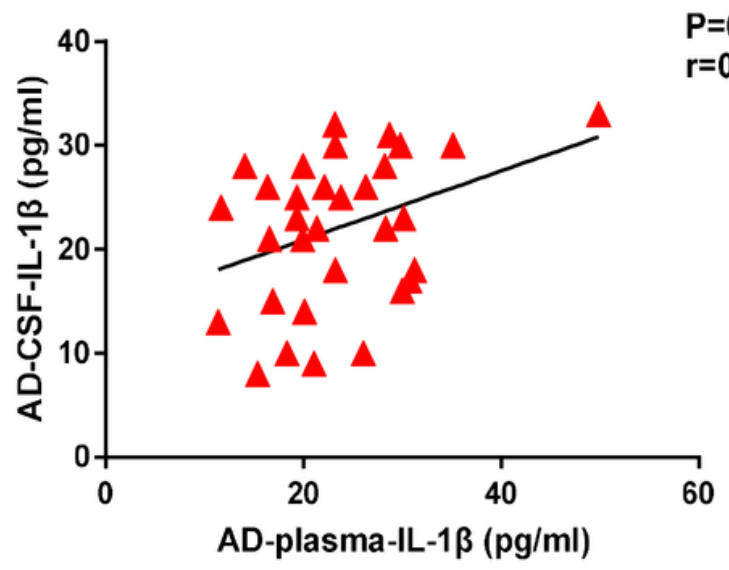

AD

\section{Figure 6}

The correlation between IL-1 $\beta$ levels in the plasma and CSF in patients with aMCI and or AD. (a) Correlation of IL-1 $\beta$ levels in the plasma and CSF in patients with aMCl. (b) Correlation of IL-1 $\beta$ levels in the plasma and CSF in patients with AD. The correlation was established by calculation of correlation coefficients. 


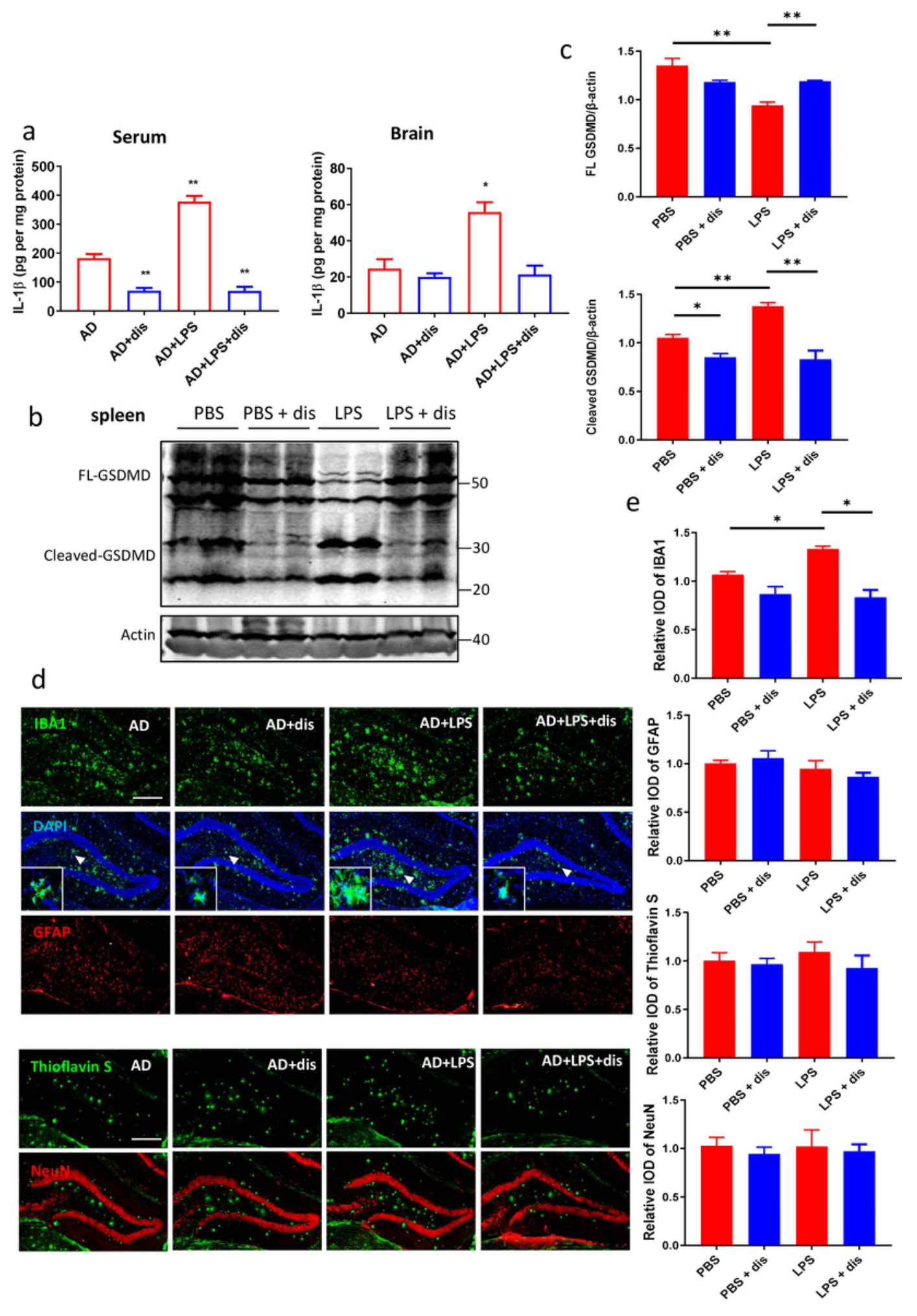

Figure 7

Peripheral pyroptosis affects the neuropathology of $5 \times$ FAD mice. (a) ELISA analysis of IL-1 $\beta$ levels in the serum and brain in the indicated mice. (b-c) Western blot analysis of the expression and activation of GSDMD in the spleen in the indicated mice. And statistics of quantified relative protein expression. (d-e) Immunofluorescence analysis of microglia (IBA1), astrocytes (GFAP), A $\beta$ plaques (Thioflavin S), and 
neurons (NeuN). And statistics of quantified relative IOD value from $\mathrm{d} .{ }^{*} \mathrm{P}<0.05,{ }^{*} \mathrm{P}<0.01$. Data are expressed as means \pm SEM $(n=3)$. Unpaired $t$-test for $a, c$ and $e$.

\section{Supplementary Files}

This is a list of supplementary files associated with this preprint. Click to download.

- table1.pdf 